\title{
eJRIEPS
}

Ejournal de la recherche sur l'intervention en éducation physique et sport

Numéro spécial 1 | 2018

Comment penser les articulations entre approches

didactiques et ergonomiques pour étudier les

pratiques d'enseignement et de formation?

\section{Gestes de contextualisation et inégalités d'apprentissage en milieu difficile}

Contextualization gestures and learning inequalities in disadvantaged

environment

Marie-Paule Poggi et Gilles Marrot

\section{OpenEdition}

Journals

Édition électronique

URL : http://journals.openedition.org/ejrieps/302

DOI : 10.4000 /ejrieps.302

ISSN : 2105-0821

Éditeur

ELLIADD

\section{Référence électronique}

Marie-Paule Poggi et Gilles Marrot, « Gestes de contextualisation et inégalités d'apprentissage en milieu difficile », eJRIEPS [En ligne], Numéro spécial 1 | 2018, mis en ligne le 01 avril 2018, consulté le 01 août 2019. URL : http://journals.openedition.org/ejrieps/302; DOI : 10.4000/ejrieps.302

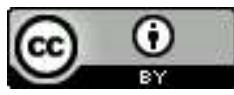

La revue eJRIEPS est mise à disposition selon les termes de la Creative Commons Attribution 4.0 International License. 
Marie-Paule Poggi et Gilles Marrot

Laboratoire CRREF (EA 4538), Université des Antilles, France

\section{Résumé}

Cette étude s'intéresse aux déterminants de l'activité didactique de l'enseignant appréhendés dans linteraction entre processus internes et externes à la classe en vue d'apprécier les effets différenciateurs, voire potentiellement inégalitaires des pratiques. Elle interroge les liens entre contexte et situation en réfléchissant à l'intérêt de mobiliser différents éclairages scientifiques tels que la sociologie, la didactique et l'ergonomie qui ne sera mobilisée qu'en termes de perspectives. Les données relèvent d'une analyse comparative de la première leçon de tennis de table de deux enseignants, un homme et une femme, exerçant en milieu difficile. On observe que les contenus enseignés, les gestes de contextualisation déployés ainsi que les effets de contexte engendrés sont le produit d'un double processus; d'une part, les caractéristiques de la situation liées au profil particulier des élèves en milieu difficile contraignent l'enseignant dans ses intentions, ses modalités d'intervention ainsi que les choix de contenus mis à l'étude, mais d'autre part, dans le curriculum en actes, professeur et élèves, de façon co-construite, réinterprètent et exploitent à leur manière les contraintes qui pèsent sur eux, cette dynamique de l'action didactique étant susceptibles d'accroitre ou au contraire de limiter les inégalités d'accès au savoir.

Mots clés : socio-didactique, inégalités, savoir, activité didactique, milieu difficile

\section{Introduction}

Cette étude s'intéresse aux déterminants de l'activité didactique de l'enseignant appréhendés dans l'interaction entre processus internes et externes à la classe en vue d'apprécier les effets différenciateurs, voire potentiellement inégalitaires des pratiques. Elle interroge les liens entre contexte et situation en réfléchissant à l'intérêt de mobiliser différents éclairages scientifiques tels que la sociologie, la didactique et l'ergonomie qui ne sera mobilisée qu'en termes de perspectives. Si l'approche ergonomique tente de comprendre l'activité dans ses liens avec la subjectivité et le pouvoir d'agir de l'enseignant sans ignorer son ancrage socio-culturel et ce qui relève d'une culture collective et 


\section{eJRIEPS Numéro Spécial n¹ Avril 2018}

historique, la didactique, elle, appréhende l'activité par le biais des préoccupations liées aux savoirs et à leur co construction en situation sans délaisser le lien avec les pratiques culturelles qui les légitiment. Enfin la sociologie questionne ce qui lie activité et processus de production des inégalités sans pour autant sous-estimer ce qui se joue à l'échelle des interactions didactiques. Ces trois approches ont en commun de questionner les liens entre le passé et le présent de l'interaction et donc les relations entre les différents niveaux de contextes mobilisés par l'enseignant en situation. Ce qui nous conduira à questionner les rapports entre contexte et situation, le contexte n'étant plus considéré comme une notion « de nature à imposer, conditionner, configurer les situations et donc à déterminer les actions (en particulier d'ordre didactique) à mener » (Castelloti, 2014, p. 118) mais plutôt comme une « notion qui contribuerait à réfléchir les situations et à en proposer des interprétations ". C'est la raison pour laquelle nous parlerons de processus de contextualisation définis comme «l'ensemble des relations interactives entre l'enseignant et le contexte en cours d'action» (Marcel, 2002), ceci nous permettra d'interroger aussi bien la place du contexte que le rôle des acteurs au sein de la situation d'enseignement apprentissage. Nous nous intéresserons aux gestes de contextualisation de l'enseignant, c'est-à-dire aux différentes façons mobilisées pour articuler plusieurs échelles de contexte pour comprendre et agir sur l'activité des élèves.

\section{Cadre théorique}

1. 1. Contexte et situation : les échelles de compréhension de l'interaction Pour comprendre une interaction didactique, il faut pouvoir l'appréhender selon différentes échelles d'analyse, du plus local au plus global. Son interprétation suppose d'en saisir les caractéristiques intrinsèques au plus près de son déroulement in situ (la situation) sans pour autant ignorer l'environnement structurel (le contexte) dans lequel ce dernier s'inscrit. Si la prise en compte du contexte est indispensable, pour autant, elle ne peut faire oublier qu'un certain nombre d'événements émerge de façon située. Dès lors, situation et contexte peuvent difficilement être pensés indépendamment l'un de l'autre. Le « contexte pertinent » (Lahire, 1998) de l'interaction didactique n'est à appréhender ni au niveau du cadre immédiat de l'interaction (la situation), ni au plan des contraintes externes qui pèsent sur elle (le contexte), mais dans une perspective, notamment sociodidactique qui vise à observer de quelle manière les dispositions incorporées perdurent dans la situation mais également se reconfigurent au contact des contraintes de cette 


\section{eJRIEPS Numéro Spécial n¹ Avril 2018}

dernière selon un processus de contextualisation liant de façon interactive contexte et situation.

De nombreux auteurs ont pointé les difficultés à appréhender le concept de contexte. On trouve dans la littérature différentes définitions qui vont d'un contexte totalement extérieur à l'action et qui viendrait la sur déterminer à des conceptions plus internaliste ou constructiviste. Ces dernières renvoient le contexte à une échelle individuelle et située qui émerge de la façon dont les acteurs eux-mêmes construisent leur action, ce qui fait dire à Blanchet (2016, p. 9) que « le contexte doit être pensé comme une construction et non comme une donnée ». On peut alors considérer que, si le contexte agit sur l'individu, la réciproque est également vraie. Le contexte a un impact sur les conduites individuelles et par son adaptation, l'acteur crée du contexte. Par conséquent, l'activité est à la fois contextualisée et contextualisante (Bru, 2004).

Un certain nombre d'auteurs partage cette orientation. Pour Bourdieu (1984), cette double dimension s'exprime dans une sorte de dialectique connivence / adaptation. En effet, pour l'auteur, l'interaction est le produit de l'actualisation de structures et dispositions sociales, les individus projettent dans la situation leurs propriétés sociales elles-mêmes directement en lien avec leur position dans la structure sociale ; ce qui d'ailleurs les incite à rechercher les situations de proximité entre dispositions et situation. Cependant, la nécessité de l'adaptation à de nouvelles situations, les ajustements imposés par les contraintes de la situation sont susceptibles d'engendrer des transformations durables de l'habitus. Même si cet aspect de ses travaux est moins souvent évoqué, Bourdieu (1984) insiste sur ces transformations durables de l'habitus produit des « ajustements qui sont sans cesse imposés par les nécessités de l'adaptation à des situations nouvelles et imprévues ». D'une certaine manière, la situation est « la condition permissive de l'accomplissement de l'habitus » (Bourdieu, 1984, p. 135).

De nombreux travaux insistent sur cette relation de circularité entre contexte et situation. Béguin et Clot (2004) posent comme condition d'une construction d'une théorie de la pratique la compréhension des liens entre le « donné et le créé ». Sensevy (2009) défend l'idée d'une «dialectique entre sens construit en contexte et sens préexistant au contexte». Cicourel (1979) observe que les «compétences interactionnelles» des acteurs sociaux, cible de ses travaux, s'exercent toujours dans un espace de contraintes structurelles qui les limitent plus ou moins. Enfin comme le rappelle Giddens (1987), l'acteur vit «le structurel dans sa « dualité » c'est-à-dire comme toujours à la fois contraignant et habilitant » (Giddens, 1987, p. 226). 


\section{eJRIEPS Numéro Spécial n¹ Avril 2018}

Contexte et situation offrent à l'acteur des opportunités d'exercer des compétences d'ajustement, d'adaptation, de contextualisation qui sont à la fois le produit de contraintes contextuelles et d'un passé incorporé mais aussi de créations singulières qui s'émancipent de ces contraintes du fait de la réflexivité, de l'intentionnalité ou encore de la rationalité stratégique des acteurs. En ce sens, ces deux concepts constituent des points d'ancrage pertinents à une analyse conjuguant sociologie, didactique et ergonomie. Nous retiendrons tout particulièrement la théorie de l'action défendue par Lahire (2008, 2012) qui conjugue modèle dispositionnaliste et contextualiste. Le dispositionnalisme est une approche qui tient compte du passé incorporé, produit d'une socialisation passée plus ou moins homogène, pour rendre intelligibles les pratiques, c'est-à-dire pour comprendre et expliquer ce qui fait agir. Ainsi l'acteur dispose d'un ensemble de dispositions incorporées mais également d'une pluralité de logiques d'action lui permettant de s'ajuster, dans certaines limites, à la diversité des situations qu'il rencontre. Par ailleurs, le contexte constitue un espace d'activation ou d'inhibition de dispositions incorporées ou encore un cadre socialisateur propice à la fabrication de nouvelles dispositions. La situation est bien le lieu d'une rencontre entre des expériences socialisatrices antérieures qui se cristallisent sous la forme de dispositions plus ou moins durables à croire, à agir et à penser et un contexte présent de l'action offrant des occasions d'expression, de neutralisation ou au encore de transformations de ces dispositions.

Prenant appui sur ces analyses, nous pouvons considérer la situation didactique comme un espace d'activation, d'inhibition ou de fabrication de nouvelles dispositions en lien avec les caractéristiques du contexte d'action. Elle se caractérise par un mouvement dynamique entre actualisation en situation des contraintes contextuelles (dispositions incorporées des enseignants et des élèves) et émergence en situation de nouvelles marges d'action. Ainsi l'enseignant produit des gestes de contextualisation qui intègrent ces différents niveaux de contexte. Notre projet est de comprendre comment l'enseignant mobilise ces derniers dans le tissage concret des pratiques.

\section{2. Contextualisation, gestes de contextualisation et fonctions didactiques}

Le contexte ne peut donc pas se trouver en simple «position explicative directe » (Raynaud, 2006). II ne représente pas une réalité extérieure à la situation mais bien un élément d'un processus de contextualisation qui organise les relations interactives entre l'enseignant et la situation et par lesquels l'individu construit son milieu (Bru, 1991). II s'agit donc bien de s'intéresser à des opérations de contextualisation et non à des contextes figés (Lahire, 2012). La contextualisation a été longuement définie dans la 
eJRIEPS Numéro Spécial n¹ Avril 2018

littérature sociologique (Lahire, 2012) mais également didactique, «prise en compte active des contextes dans le tissage concret des pratiques didactiques » selon Blanchet (2009), « ensemble de processus transpositionnels » pour Delcroix, Forissier et Anciaux (2013) ou encore « ensemble des relations interactives entre l'enseignant et le contexte en cours d'action » d'après Marcel (2002, p. 104). Tous ces auteurs s'accordent pour dire que la contextualisation s'apparente à un processus qui aide à mieux comprendre les liens dynamiques entre contraintes contextuelles et action en situation, entre action conjointe de l'enseignant et des élèves et les éléments de contexte.

Ce processus de contextualisation s'opérationnalise dans des gestes de contextualisation qui prennent en considération la diversité des contextes. BrièreGuénoun et Musard (2012) en font une caractéristique première des gestes professionnels qu'elles décrivent comme « ancrés dans les pratiques » et révélateurs des « façons dont l'enseignant définit, interprète et s'adapte au contexte pour concevoir et conduire son enseignement in situ ». Selon les auteures, ces derniers actualisent les préoccupations, les valeurs, les expériences et les savoirs de l'enseignant en lien avec les contraintes contextuelles et l'activité d'apprentissage des élèves. Ces gestes de contextualisation se déploient en combinant et articulant différentes strates contextuelles qui relèvent d'une échelle intime, subjective (référence à ce que l'élève pense, ressent personnellement), une échelle situationnelle (référence à ce à ce qui se vit en classe), une échelle contextuelle (référence à ce qui est externe à la classe et à l'élève), une échelle dispositionnelle (référence à ce qui externe à la classe et propre à l'élève).

Au final, on peut dire que le travail de contextualisation de l'enseignant relève d'une compétence à articuler ces différentes échelles de contexte pour comprendre et agir sur l'activité des élèves, d'une certaine manière il s'agit d'une compétence à jouer sur les conditions de possibilité de la situation d'enseignement pour construire cette dernière. II révèle les façons selon lesquelles les données contextuelles antérieures à l'action sont reconfigurées, interprétées, traitées en situation vécue. Le processus de contextualisation relève de la façon dont les variables contextuelles externes sont traitées dans et par la pratique (Bru, 1991).

Pour résumer, on peut dire que la contextualisation, et les gestes de contextualisation qui l'opérationnalisent et en permettent l'expression, répond à la volonté de prendre des distances par rapport à une conception qui placerait le contexte en position explicative surplombante et externe laissant penser qu'un certain nombre de normes, institutionnelles et sociales, s'imposeraient de façon mécanique en situation. II s'agit alors d'observer quels 


\section{eJRIEPS Numéro Spécial n¹ Avril 2018}

processus de définition et d'ajustements de principes, de démarches et de contenus se déploient en classe via la mise en œuvre de gestes de contextualisation.

Nous avons procédé à un recueil des gestes professionnels en situation à partir de deux critères: les échelles de contexte mobilisés et les fonctions didactiques qui leur sont associées. Ces dernières permettent d'identifier de quelles façons se jouent le jeu didactique à ces différentes échelles de contexte. Nous nous sommes appuyés sur la typologie élaborée par Sensevy (2006) qui définit quatre catégories de gestes de l'enseignant en lien avec leurs fonctions: définir (initier le jeu), dévoluer (créer les conditions pour que l'élève accepte de jouer), réguler (faire agir les élèves) et institutionnaliser (fixer les manières de faire et de penser légitimes).

\section{Problématique}

Nous cherchons à décrire, comprendre et expliquer la prise en compte par l'enseignant, dans les situations de classe, des variables subjectives, contextuelles et situationnelles et observer leur impact sur l'organisation des modalités d'enseignement et les inégalités d'apprentissage auxquels sont soumis les élèves. Plus précisément, nous tenterons de comprendre comment l'enseignant actualise en situation, via ses gestes de contextualisation, les différentes échelles de contexte. Nous pourrons apprécier le degré de contextualisation de la démarche d'enseignement dans un contexte particulier, le milieu difficile en mobilisant le discours sur les pratiques (tenus par les enseignants euxmêmes via les entretiens) et l'observation des pratiques (via les vidéos).

\section{Méthodologie}

3. 1. Les modalités de recueil

Les données relèvent d'une analyse comparative de la première leçon de tennis de table de deux enseignants, un homme et une femme, exerçant en milieu difficile. Nous avons filmé les leçons et procédé à un entretien semi-directif ante et à un entretien post d'autoconfrontation. Le dispositif nous permet d'appréhender les aspects contextuels qui sont mobilisés par les acteurs eux-mêmes dans leurs conversations et les implicites qui ne sont pas signalés par eux afin d'accéder à une compréhension didactique et sociologique de ce qui se passe dans cette interaction. Cette démarche nous semble être en mesure de croiser l'interne et l'externe des situations observées, d'appréhender l'enchevêtrement des différents niveaux de contexte et de penser les pratiques enseignantes et des élèves à 


\section{eJRIEPS Numéro Spécial n¹ Avril 2018}

l'articulation entre dispositions (propriétés sociales incorporées des acteurs, enseignant et élèves), contextes (propriétés sociales objectivées, système de normes et contraintes) et milieu didactique (tout ce qui agit sur l'élève ou / et ce sur quoi l'élève agit).

Le dispositif est classique, il met en tension trois types de traces: (1) les pratiques enseignantes et des élèves telles qu'elles se mettent en œuvre en situation de classe (données vidéo), (2) les déclarations des enseignants (entretien ante et post d'autoconfrontation) en lien avec (3) l'analyse des savoirs en jeu dans les transactions didactiques (analyse a priori).

\section{2. Le traitement}

Le traitement des données a suivi trois étapes. Nous procédons d'abord à une analyse extrinsèque au cours de laquelle, à l'appui des vidéos, nous avons observé ce que disent et font enseignant et élèves. Puis, nous soumettons les entretiens à une analyse de contenu afin d'accéder à cette part de signification non conscientisable par l'acteur de son activité que le chercheur a pour mission de reconstruire à l'aide des différentes traces recueillies. Enfin, nous terminons par la mise en lien des deux traces pour comprendre et identifier le rôle des différents déterminants de l'action didactique. Dans cette dernière phase, l'analyse des données a conduit à repérer un certain nombre d'indicateurs de contextualisation (figure 1) repérables soit dans les vidéos de leçons, soit dans les discours d'entretiens. Ces indicateurs ont été construits à trois niveaux : les pratiques, les principes et les savoirs, chacun de ces niveaux permettant de décrire et comprendre l'articulation entre contexte et situation constitutive des processus de contextualisation. Les données étant en cours de traitement, la catégorisation proposée est sujette à évolution. 


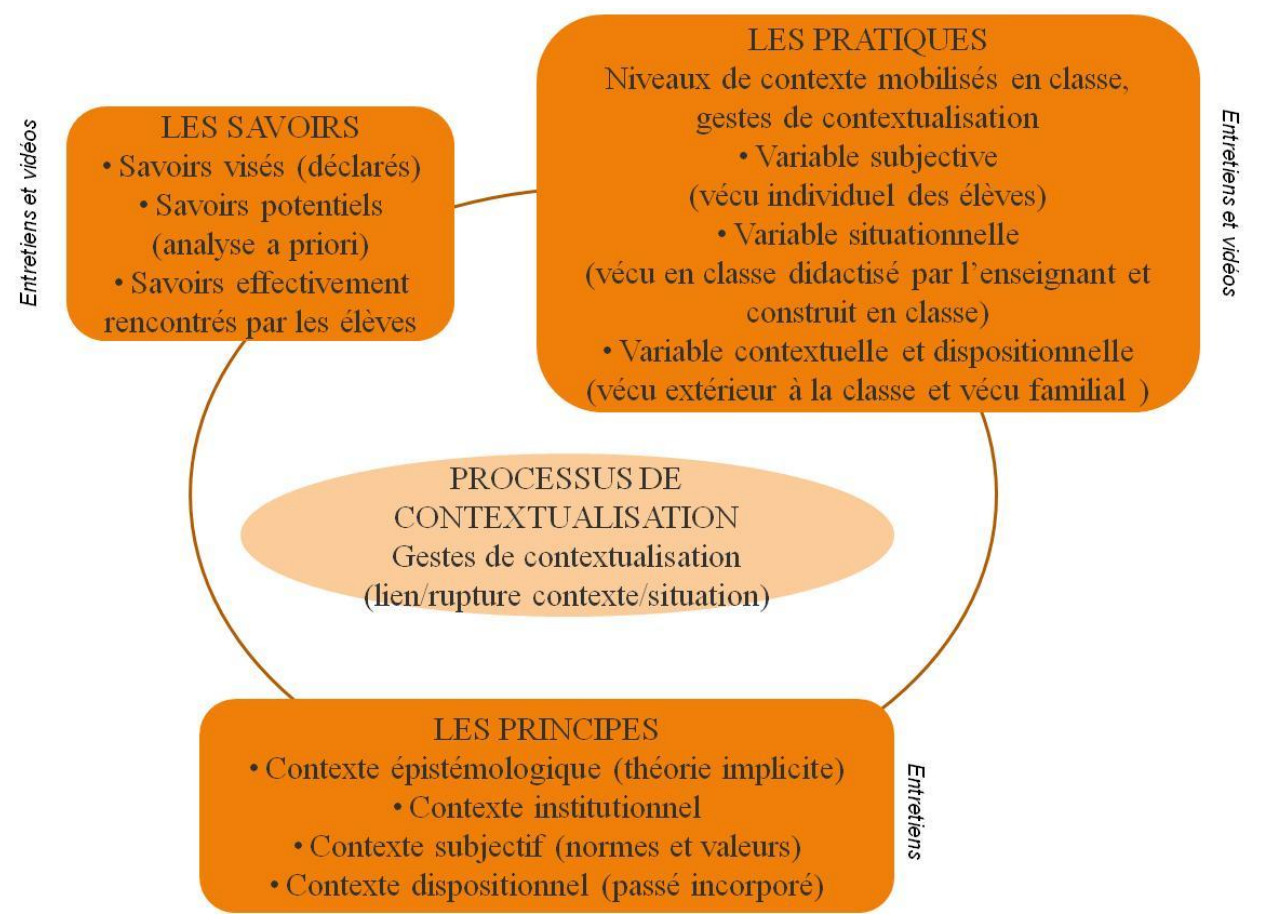

Figure 1. Modélisation des indicateurs du processus de contextualisation.

Nous recherchons dans l'interaction des " indices de contextualisation " c'est-à-dire tout ce qui peut fournir des informations sur les interactions entre les différents niveaux de contexte (ou strates contextuelles) et leurs effets sur les inégalités d'apprentissage. En fait, nous voulons comprendre comment la «capacité de l'enseignant à intégrer différentes sphères contextuelles " (Tupin et Sauvage, 2010) influe sur le processus enseignement apprentissage.

3. 3. Le terrain d'investigation retenu : le milieu difficile

Cette étude prend pour objet d'étude l'activité du professeur d'EPS en milieu difficile, c'est-à-dire dans un espace de travail rude et complexe, soumis à des contraintes qui testent les limites de sa "capacité d'affecter son milieu par son initiative " (Clot, 2013). On compte aujourd'hui un nombre conséquent de travaux consacrés à l'enseignement en milieu difficile, au sein de ce que Van Zanten appelle "l'école de la périphérie " (Van Zanten, 2001). Les travaux menés dans le champ de la didactique, tout comme ceux menés dans le champ de la sociologie (Charlot, Bautier, Rochex, 1992 ; Rochex, 1995 ; Van Zanten, 2001), soulignent : un éclatement et une réduction des contenus enseignés dans les établissements scolaires dits difficiles ainsi qu'une tendance à valoriser des savoirs plus superficiels et des savoirs-faire sociaux (Amade-Escot \& Venturini, 2009 ; Monnier \& Amade-Escot, 2009). On note un effet de sur-ajustement des tâches aux 


\section{eJRIEPS Numéro Spécial n¹ Avril 2018}

caractéristiques des élèves, des difficultés à enrôler ces derniers dans de réels enjeux d'apprentissage au-delà de la simple effectuation des tâches ou encore une révision à la baisse des contenus des programmes visés.

Par ailleurs, les situations de travail proposées ainsi que les modes d'ajustements des enseignants aux caractéristiques des élèves (sur ou sous ajustement didactique) entravent le travail de secondarisation et de décontextualisation des élèves en brouillant les enjeux cognitifs et langagiers des tâches (Bautier \& Goigoux, 2004 ; Bautier \& Rayou, 2009 ; Rochex \& Crinon, 2011). Par exemple, l'habillage ludique des tâches en prise avec l'expérience familière des élèves, les situations à faible cadrage ou encore la pédagogie du « faire » sont à l'origine de graves malentendus sur la nature de l'activité intellectuelle sollicitée par la tâche et pertinente pour apprendre. Ces dispositifs alimentent des processus de différenciation «passive» (Rochex \& Crinon, 2011) qui laissent dans l'implicite les activités requises pour réussir, condamnant à l'échec les élèves les moins préparés par leur milieu familial à décoder ces sous entendus.

Les recherches en didactique pointent une tendance à simplifier, éparpiller et décontextualiser les tâches en milieu difficile provoquant un appauvrissement des apprentissages et un « évanouissement du savoir » (Marlot \& Toullec-Théry, 2011). Par ailleurs, la diminution des phases collectives d'institutionnalisation des savoirs nuit à la constitution d'une mémoire didactique et les formes de régulation "pragmatiques» (réussir) prennent le pas sur les « régulations épistémiques » (comprendre) limitant ainsi l'activité de conceptualisation (Bocchi, 2012).

Tous ces travaux ont en commun de s'intéresser à la spécificité de la nature des savoirs transmis et aux modalités de leur transmission en ciblant des grains d'analyse variés selon différentes problématiques de recherche. Elles conduisent à penser le milieu difficile comme un environnement spécifique qui agit un peu comme une loupe posée sur la question des interactions en contexte et sur l'étude de leurs effets sur la situation enseignement apprentissage. En effet, les pratiques d'enseignement en établissements défavorisés offrent un véritable laboratoire, en situation scolaire authentique (Kherroubi et Rochex, 2004), de pratiques d'adaptation, de différenciation, d'ajustement aux caractéristiques des publics scolaires et plus particulièrement à des élèves bien souvent en décalage, voire en rupture, avec les normes et les exigences scolaires traditionnelles. 


\section{eJRIEPS Numéro Spécial n¹ Avril 2018}

\section{Résultats}

Les résultats n'étant pas traités dans leur totalité, nous ne pourrons évoquer que quelques pistes. Nos observations mettent en évidence l'apparition de gestes professionnels de contextualisation ainsi que l'émergence « d'effets de contexte » qui s'accompagne d'un impact différenciateur sur les conduites des élèves.

\section{1. Echelles de contexte mobilisés}

Nous n'avons pas pu, à ce stade d'avancée de la recherche, appréhender les processus de contextualisation à l'aide de tous les indicateurs identifiés dans la figure 1. Dans le cadre de cet article nous avons essentiellement privilégié le niveau des pratiques en ignorant pour l'instant le lien avec les savoirs (analyse a priori des tâches) et partiellement avec les principes (entretiens ante et post). Cette partie non explorée du travail s'inscrit dans des perspectives de recherche à venir. Par ailleurs, seule la première leçon a été soumise à un traitement méticuleux des données; par conséquent, la taille de l'échantillon appelle évidemment à la plus grande prudence quant aux conclusions qui seront proposées.

Nous avons procédé à un recueil des gestes de contextualisation en situation à partir de deux critères: les échelles de contexte mobilisées et les fonctions didactiques qui leur sont associées.

Le recours à l'échelle contextuelle vise principalement à normaliser l'activité des élèves en rappelant les règles qui gèrent la situation didactique comme par exemple les règles de vie, les conseils techniques, la gestion du matériel, les contraintes d'évaluation ou encore les préconisations tactiques, comme par exemple, lorsque l'enseignant précise « ta raquette c'est vraiment le prolongement de ta main, j'ouvre pouce index, j'ai un doigt derrière pour pouvoir vraiment jouer avec mon poignet (geste à l'appui) » ou encore lorsque l'enseignante affirme "les déplacements en tennis de table c'est ça, je suis centrée par rapport à la table, (en montrant) coup droit ici, revers, je me déplace j'attaque ». Dans ce cadre, c'est bien la pratique sociale tennis de table qui sert de référence et qui est présentée aux élèves, dans un discours universalisant, sous la forme d'un ensemble de recommandations incontestables. La mobilisation de cet espace sert principalement les fonctions de régulation et d'institutionnalisation.

Lorsque l'enseignant se réfère à l'échelle situationnelle, vécu en classe, c'est principalement pour organiser le milieu à des fins de régulation et de définition. L'enseignant présente ou rappelle ce qu'il y a à faire, s'assure de l'avancée des apprentissages ou encore vérifie la progression dans les exercices. Dans ce cas de 


\section{eJRIEPS Numéro Spécial n¹ Avril 2018}

figure, le projet est de réguler ou de définir comme lorsque l'enseignant assure « Tu vois ta raquette la au moment où tu frappes elle est comme ça (enseignant montre le tamis orienté vers le haut), voilà là c'est mieux, j'aime mieux ça même si ça va dans le filet ».

Enfin, l'intrusion dans l'échelle subjective, relative à l'expérience personnelle de l'élève considérée dans sa singularité, permet à l'enseignant de s'assurer que les élèves comprennent, de les encourager à pérenniser ou reprendre l'activité, de les rassurer ou encore de dédramatiser les difficultés comme par exemple lorsque l'enseignante lance en début de leçon « on essaye, c'est la première séance, c'est pas très grave si on n'y arrive pas » ou lorsque l'enseignant signifie à un élève, Alia, qui déclare ne pas aimer l'exercice proposé, «c'est pas parce qu'on aime pas qu'on ne fait pas Alia, on ne fait pas que ce qu'on aime dans la vie Alia, t'es d'accord avec moi on fait pas toujours que ce qu'on aime, si? Tu fais toujours que ce que tu aimes toi dans la vie (Alia répond oui), ça m'étonnerait ».

Au cours de la leçon, les enseignants pianotent sur ces différentes échelles de contexte. Sans surprise, c'est le niveau de la classe qui est d'abord mobilisé $(50,6 \%)^{1}$ puis le contexte hors de la classe $(28,2 \%)$, puis l'échelle subjective et intime de l'élève $(20,8)$, le dispositionnel étant peu évoqué $(0,4 \%)$. Comme on pouvait s'y attendre, l'échelle situationnelle structure majoritairement les interactions en classe pour les deux enseignants.

En revanche, l'enseignante fait davantage de place à l'échelle contextuelle (7 points séparent les deux intervenants) tandis que l'enseignant privilégie l'espace subjectif (6 points de différence).

Quelle que soit l'échelle de contexte mobilisée, l'acte de régulation (64,9\%), et dans une moindre mesure de définition $(18,9 \%)$, structure fondamentalement l'action didactique laissant peu de place à l'institutionnalisation $(8,5 \%)$ ou la dévolution $(7,7 \%)$. Cependant, les deux enseignants se différencient avec une prédilection pour l'institutionnalisation en ce qui concerne l'enseignante et pour la définition du côté de l'enseignant. Les actes de dévolution sont peu fréquents pour l'un comme pour l'autre.

L'échelle contextuelle est mobilisée principalement à des fins de régulation par les deux enseignants mais également d'institutionnalisation pour l'enseignante. L'échelle situationnelle est mise au service de la régulation pour l'enseignante et à part égale de la

\footnotetext{
${ }^{1}$ ces résultats ont été établis à partir d'une analyse de contenu exhaustive des verbatims des deux leçons.
} 
eJRIEPS Numéro Spécial n¹ Avril 2018

définition et de la régulation pour l'enseignant. Enfin, l'échelle subjective est exclusivement réservée à la régulation.

\section{2. Variations selon les enseignants}

En ce qui concerne l'enseignante, la référence majeure (31,3\% des unités de sens) à un espace culturel extérieur à la classe sert de point d'appui à un travail de régulation et d'institutionnalisation. Cette dimension impersonnelle, se référant à des assujettissements extérieurs à la classe liés fréquemment à des rappels réglementaires et techniques spécifiques à la pratique sociale de référence, constitue le socle dominant de l'intervention de l'enseignante lui donnant ainsi une tonalité contextualisée marquée.

La place octroyée à l'espace situationnel se manifeste sous la forme de commentaires et d'appréciations portées sur l'activité de l'élève, principalement au cours d'interactions individuelles visant à faire agir les élèves et à organiser le milieu matériel (actes de régulation).

Enfin, sans pour autant la négliger, l'enseignante ne donne pas la priorité à l'échelle subjective $(18,4 \%)$. Elle se manifeste sous la forme d'interventions courtes, dans le cadre de régulation individuelle, à forte tonalité affective. L'enseignante l'utilise pour rassurer, encourager les élèves ou encore dédramatiser une situation tendue en manipulant à doses homéopathiques humour et dérision.

De son côté, l'enseignant couple de façon parfaitement équilibrée échelle contextuelle et subjective comme si la référence extérieure ne pouvait impacter l'activité de l'élève que référée à l'espace intime de ce dernier. Le vécu individuel des élèves est appréhendé principalement sous la forme d'encouragements à comprendre, réfléchir et faire, de réassurance (croire que c'est possible), de réprimande. Cette incursion dans l'espace intime des élèves prend la forme de plaisanterie ou d'incitation à penser son rapport au travail et à l'engagement dans la tâche (comme par exemple : « c'est pas parce qu'on aime pas qu'on ne fait pas Alia, on ne fait pas que ce qu'on aime dans la vie Alia, t'es d'accord avec moi on fait pas toujours que ce qu'on aime, si ? Tu fais toujours que ce que tu aimes toi dans la vie (Alia : oui) ça m'étonnerait. »).

Comme précédemment, l'espace situationnel reste privilégié. On observe que ce registre situationnel se manifeste par un équilibre entre régulation et définition. En situation, l'enseignant consacre autant de temps à poser les conditions pour initier le jeu qu'à s'assurer du bon déroulement du jeu. 


\section{eJRIEPS Numéro Spécial n¹ Avril 2018}

\section{Discussion}

Pour contextualiser leur pratique, les enseignants déploient des gestes professionnels de contextualisation communs et spécifiques. A population scolaire similaire, on note des éléments de généricité mais également de spécificité.

5. 1. Des éléments de généricité, des points communs entre les deux enseignants

L'analyse des gestes de contextualisation déployés par nos deux enseignants conduit à penser leur activité didactique comme à la fois contextualisée, c'est-à-dire impactée par les assujettissements de l'espace contextuel, mais également contextualisante car inscrite dans les registres situationnel et subjectif d'action.

La référence à l'externe de la classe s'exprime principalement via la référence fréquente aux exigences de la pratique sociale de référence. Pour les deux enseignants, la référence à la pratique sociale joue un rôle déterminant dans la mise en acte des contenus enseignés. Cette dernière constitue un cadre qui borne de façon claire la définition de ce qu'il y a à enseigner et apprendre. En entretien, les enseignants déclarent que tout semble possible en matière d'ajustement et d'adaptation en situation à condition que les élèves « restent dans l'activité ». Même si cette référence à cet espace externe à la classe pèse lourd dans la façon de mettre en œuvre l'interaction didactique, d'autres déterminants externes en revanche semblent ignorés. Par exemple, en entretien, à aucun moment, les prescriptions institutionnelles n'apparaissent comme un cadre de référence sur lequel l'enseignant pourrait ou devrait s'appuyer. Nous avons affaire à des intervenants chevronnés dont l'expertise autorise peut-être une certaine liberté à l'égard des injonctions institutionnelles.

Second constat, quelle que soit l'échelle de contexte mobilisée, c'est bien l'activité de régulation, et secondairement de définition, qui organise l'activité de l'enseignant au détriment des activités d'institutionnalisation et de dévolution. Rappelons que nous n'avons traité que les données de la première leçon ce qui peut expliquer cette tendance. Cependant, on retrouve des constats déjà observés par d'autres comme Bautier (2015) qui a mis en évidence que dans de nombreuses classes les formes d'activités proposées aux élèves conduisent à la « quasi-disparition d'un discours d'enseignement ». Comme Bautier, nous pouvons constater que «les échanges s'individualisent, portent sur le commentaire de ce que fait tel ou tel élève, sans renvoyer à un moment ou à un échange collectif » (p. 21). 


\section{eJRIEPS Numéro Spécial n¹ Avril 2018}

II nous reste à étudier et comprendre avec davantage de finesse quelle est la nature des interactions entre l'impersonnel du contextuel et le personnel du subjectif au sein même de la classe. Le recours à l'espace subjectif vient souvent ponctuer une série d'injonctions contextuelles liées au respect du règlement ou à une exigence de conformité à la technique. Il apparaît donc comme un moyen d'informer l'élève qu'il dispose d'une marge de manœuvre de l'élève à l'intérieur toutefois d'un espace strictement balisé. Par exemple, l'enseignant s'adressant à un élève déclare «c'est pas grave, c'est pas grave mais tu n'as pas le droit de toucher la table, tu es toujours appuyé dessus. Je te le dis pour la suite », discours qui à la fois et dans un même moment libère et rassure mais qui également contraint.

\section{2. Des gestes de contextualisation spécifiques}

Pour contextualiser leur pratique, les enseignants déploient des gestes professionnels de contextualisation qui leur sont propres. A population scolaire similaire, chacun contextualise à sa manière. Nous constatons que les liens entre l'externe, le subjectif et le situationnel ne sont pas mécaniques et interagissent de façon différente selon l'enseignant concerné.

L'enseignant mise tout particulièrement sur un équilibre entre interne et externe à la situation pour ajuster son intervention auprès des élèves. La référence à l'espace intime de l'élève constitue un point d'appui pour rappeler à ce dernier que, dans le cadre des contraintes qui lui est imposé, il dispose d'une marge de manœuvre dont il peut et doit s'emparer pour s'approprier les contenus d'enseignement comme le montre cet échange : (s'adressant à un élève) « oriente plus ta raquette vers la table... (l'enseignant compte à haute voix les échanges dont l'objectif est de durer) ça fait un, on recommence, plus doucement voilà, 1 bien, 2 voilà, 3 (rupture de l'échange)... pourquoi tu viens prendre en revers à droite ? C'est ça qui te fout dedans sinon c'était bien...3, tu vois, donc c'est possible ». De façon différente, l'incursion dans l'espace intime mise en œuvre par l'enseignante relève davantage de stratégies de dédramatisation de l'échec, moins en lien avec les contenus enseignés, visant, par le recours à la plaisanterie ou à une certaine familiarité avec les élèves, à désamorcer certains blocages ou conflit avec les élèves car comme elle le précise en entretien, « c'est des élèves avec qui on est toujours obligé de négocier ».

Autre différence notable entre nos deux intervenants, les stratégies de contextualisation reposent pour l'un sur la régulation et la définition, et pour l'autre, l'enseignante, sur la régulation et l'institutionnalisation. L'enseignante pose d'emblée, dès la première leçon, 


\section{eJRIEPS Numéro Spécial n¹ Avril 2018}

les contenus légitimes à s'approprier. Son collègue insiste davantage sur les règles définitoires (Sensevy, 2006) du jeu didactique à partir desquels il va amener à avancer.

\section{3. Conséquences différenciatrices sur les élèves et effets de contexte}

On observe également des " effets de contexte ». A l'instar des effets de contrat mis en exergue par Brousseau (1998), nous pouvons constater l'apparition d'effets de contexte tels que définis par Delcroix, Forissier, Anciaux (2013) : « Ces effets se manifestent par un décalage entre un objectif d'enseignement ou d'apprentissage et sa réalisation, et seront qualifiés d'effets de contexte quand ce décalage est attribuable aux différents contextes en présence dans le processus didactique » (p. 142) autrement dit à l'entrecroisement en situation des variables subjectives, situationnelles et contextuelles. Comme le souligne Brousseau, porté par le désir de voir ses élèves réussir, l'enseignant, en cas d'échec répété, tend à négocier à la baisse les exigences du contrat didactique. Sous l'emprise du processus de contextualisation, il tend à réduire les exigences d'apprentissage.

De fait, on observe une contextualisation qui, par certains côtés, est susceptible de détourner les élèves des savoirs. Par exemple, l'enseignante, face à la difficulté persistante de certains élèves, réaffirme à plusieurs reprises que le résultat lui importe peu pourvu que les élèves restent engagés dans la tâche. Elle réévalue délibérément à la baisse les contenus à s'approprier au profit d'une préservation d'un climat de classe agréable. Elle le manifeste aussi bien en entretien que dans son discours dans le cours même de la leçon. Le système des savoirs transmis subit donc des variations potentiellement propices à la fabrication d'inégalités. Ce risque est accentué par une anticipation sur l'intérêt probable des élèves, anticipation qui provoque une limitation des objectifs visés par l'enseignante. On peut faire l'hypothèse que cette « privation de savoirs » (Tanguy et al., 1984) par anticipation engendre un accès inégal des élèves à la culture scolaire (au moins celle légitimée par les textes officiels) et incite implicitement les élèves à se conformer à des exigences moindres en termes d'acquisition de savoirs (comme le confirment les travaux sur les « effets d'attente »). En situation, on peut donc conclure à l'effet inégalitaire de l'emprise de variables dispositionnelles sur les pratiques comme sur le discours de l'enseignante.

L'enseignant procède un peu différemment en situation. A partir d'une définition systématiquement organisée, il incite les élèves à expérimenter des réponses d'action. Le savoir enseigné est ensuite déclaré, nommé, institué par l'enseignant puis déployé de façon interactive avec les élèves sous la forme d'un jeu de questions-réponses (cet aspect apparait surtout dans les leçons suivantes que nous n'avons pas analysées dans le cadre 
eJRIEPS Numéro Spécial n¹ Avril 2018

de cet article). L'analyse des données vidéo et d'entretien indique qu'il tente de mettre en œuvre des processus d'accès au savoir visant à une secondarisation (Bautier et Goigoux, 2004). On peut observer que les élèves jouent le jeu et s'impliquent pleinement dans cette situation. Comme le montrent Bautier et Goigoux (2004), les inégalités scolaires en matière d'apprentissage et d'accès aux savoirs résultent de l'opacité et du caractère implicite des attentes et des difficultés éprouvées par les élèves pour identifier les enjeux cognitifs des tâches. Pour sortir de cette impasse, les auteurs formulent l'hypothèse d'un nécessaire travail de secondarisation amenant les élèves, au-delà d'une simple logique du faire et de la réussite immédiate, à saisir ce que les tâches qui leur sont proposées leur permettent d'apprendre. Comme le soulignent les auteurs, « Aujourd'hui à l'école, il ne suffit pas de « faire ce que le maitre dit » pour réussir, il faut aussi comprendre ce qu'on fait et comment on le fait » (p. 91). On peut faire l'hypothèse que l'usage particulièrement stable que l'enseignant fait de ce type de tâche (au cours du cycle mais également d'un cycle à l'autre) est producteur chez les élèves d'une réelle activité d'apprentissage favorable à la réduction des inégalités.

Pour l'instant, un premier niveau d'observation des élèves révèle que :

- les élèves brouillent le message en faisant preuve de connivence (donnent des signes extérieurs d'écoute attentive) au moment de la définition et en développant des comportements hors tâche au moment de la mise en œuvre ;

- les «bons » expérimentent des comportements hors tâche, ils passent à côté des tâches exigeantes mais cela est considéré comme un détour nécessaire par l'enseignant (cf entretien post « je le laisse aller au bout pour qu'il comprenne » pour « qu'il prenne conscience », laisser les élèves expérimenter) et comme un moyen de gérer le dilemme entre préoccupations socialisantes et préoccupations d'apprentissage pour maintenir la relation didactique et maintenir les élèves en activité ;

- les élèves en difficulté tentent de se mettre en conformité avec les attentes de l'enseignant et suivent scrupuleusement les consignes même lorsque la tâche est dépourvue de sens pour eux ;

- tous les élèves semblent profiter des phases collectives d'institutionnalisation des savoirs étroitement articulées au temps de pratique (impliquent directement les élèves) et ritualisées.

L'état actuel d'avancement du traitement des données, ne nous permet pas d'aller plus loin dans notre analyse. 


\section{eJRIEPS Numéro Spécial n¹ Avril 2018}

\section{Conclusion}

Les analyses que nous avons présentées, qui méritent encore d'être davantage étayées, montrent la complexité et l'hétérogénéité des gestes de contextualisation qui conjuguent différentes échelles de contexte (situationnelle, contextuelle et subjective) en classe en milieu difficile. II reste désormais à examiner de façon plus précise les effets sur l'activité d'apprentissage des élèves ainsi que sur la production des inégalités entre ces derniers. Pour comprendre nos résultats, on peut faire l'hypothèse que en milieu difficile, les contenus enseignés, les gestes de contextualisation déployés ainsi que les effets de contexte engendrés sont le produit d'un double processus; d'une part, les caractéristiques de la situation liées au profil particulier des élèves en milieu difficile contraignent l'enseignant dans ses intentions, ses modalités d'intervention ainsi que les choix de contenus mis à l'étude, mais d'autre part, professeur et élèves, de façon coconstruite, réinterprètent et exploitent à leur manière les contraintes qui pèsent sur eux, cette dynamique de l'action didactique étant susceptible d'accroitre ou au contraire de limiter les inégalités d'accès au savoir.

Notre objectif était de comprendre la manière dont deux enseignants, exerçant en milieu difficile, prennent leur décision sous l'influence de différents déterminants. Dans cette perspective, nous voulions repenser la prise en compte des contextes au sein de la situation enseignement - apprentissage. A l'instar de Dolz et Tupin (2001, p. 84), il nous semblait intéressant de prendre appui sur « une tentative de re-définition de la situation conçue comme la résultante des rapports dialectiques entre, «l'ici et maintenant » de la classe - habitée par la micro-société constituée par l'enseignant et les apprenants - et « les différents cercles contextuels » qui marquent de leur empreinte les conditions d'expression du sens pratique qui relèvent d'une hybridation entre habitus individuel, habitus de classe et habitus professionnel ». Quelques pistes de résultats émergent et méritent d'être explorés davantage. L'analyse à l'échelle situationnelle révèle « des discours enseignants qui régulent plus qu'ils n'enseignent » (Bautier, 2015, p. 21), sur le plan contextuel, on observe que la référence à la pratique sociale contraint fortement le cours de l'action didactique, contrainte qu'une intervention à l'échelle subjective vise à adoucir.

Même si l'approche socio-didactique reste notre principale référence, d'autres approches pourraient être d'un grand secours pour mieux comprendre les pratiques d'enseignement - apprentissage. Par exemple, la clinique de l'activité, telle que proposée par Clot (1999), en lien avec les approches ergonomiques, met avant cette nécessité d'articuler différents 
eJRIEPS Numéro Spécial n¹ Avril 2018

niveaux de contexte pour comprendre l'activité et le métier. L'activité n'est pas seulement déterminée par «l'impersonnel de la prescription »(Clot, 2013), elle est le produit d'un rapport entre le donné générique et ce qui se produit en situation réelle, elle est à la fois adhésion à la prescription et activité personnelle, subjective et créatrice. L'activité est pensée comme le produit de «multiples contraintes simultanées et enchevêtrées » (Lahire, 1996) qu'elle contribue elle-même à produire, ce qui fait dire à Clot et Lhuilier (2012) que « l'activité n'existe dans un contexte qu'en produisant du contexte pour exister » (p. 16). Par conséquent, cette ambition de penser l'activité à la croisée de différents niveaux de contexte intéresse autant la didactique, la sociologie que l'analyse de l'activité qui peuvent probablement associer leur cadre théorique et leur méthodologie pour mieux appréhender les formes et modalités de contextualisation à l'œuvre dans la pratique enseignante. Ainsi la recherche présentée peut tenter de questionner les effets différenciateurs des pratiques dans le cadre d'une approche de type socio-didactique qui gagnerait à être articulée de façon plus fine à un éclairage ergonomique.

\section{Bibliographie}

Amade-Escot, C. \& Venturini, P. (2009). Le milieu didactique : d'une étude empirique en contexte difficile à une réflexion sur le concept, Education \& Didactique 3(1), 7- 43.

Bautier, E., \& Goigoux, R. (2004). Difficultés d'apprentissage, processus de secondarisation et pratiques enseignantes : une hypothèse relationnelle. Revue française de pédagogie, 148, 89-100.

Bautier, E. (2015). Comment l'école amplifie les inégalités sociales et migratoires ? Pratiques scolaires dominantes et inégalités sociales au sein de l'école. Rapport pour le CNESCO.

Bautier, E. \& Rayou, P. (2009). Les inégalités d'apprentissage. Programmes, pratiques et malentendus scolaires. Paris : Presses universitaires de France.

Béguin, P., Clot, Y. (2004). L'Action située dans le développement de l'activité [en ligne]. @activités, volume 1, 2. Page consultée le 15 mars 2006 de http://www.activites.org/

Blanchet, P. (2009). Contextualisation didactique : de quoi parle-t-on ? Le français à l'université, 2. En ligne http://www.bulletin.auf.org/IMG/pdf_Journal_AUF_14-23.pdf 


\section{eJRIEPS Numéro Spécial n¹ Avril 2018}

Blanchet, P. (2016). Contextualisations didactiques et didactologiques : questions en débat. Contextes et Didactiques, 7, 8-14.

Bocchi, PC. (2012). Les formes de régulation de l'activité des élèves. Recherche en éducation, Hors Série, 4, 55-67.

Bourdieu, P. (1984). Questions de sociologie. Paris : Les Editions de Minuit.

Brière-Guenoun, F. \& Musard, M. (2012). Analyse didactique des gestes professionnels d'étudiants stagiaires en éducation physique et sportive. Revue des sciences de l'éducation 38(2), 255-454.

Brousseau, G. (1998). Théorie des situations didactiques. Grenoble: La pensée sauvage.

Bru, M. (1991). Les variations didactiques dans l'organisation des conditions d'apprentissage. Toulouse : Éditions Universitaires du Sud.

Bru Marc (2004). Prise en compte du contexte dans l'étude des pratiques de formation et d'enseignement. Dans Jean-François Marcel \& Patrick Rayou (dir.). Recherches contextualisées en éducation (pp. 63-76).

Castellotti, V. (2014). Contexte, contextualisation, cultures éducatives. Quels usages ? Pour quelles orientations de la recherche en DDL ? Dans S. Babault, M. Bento, L. Le Ferrec et V. Spaeth (dir.), Actes du colloque international Contexte global et contextes locaux. Tensions, convergences et enjeux en didactique des langues (pp. 111-124). Paris : Université Paris Sorbonne/FIPF. Disponible en ligne sur http://www.academia.edu/12308739/Contexte_contextualisation_cultures_éducati ves._Quels_usages_Pour_quelles_orientations_de_la_recherche_en_DDL_.

Charlot, B., Bautier, E. \& Rochex, JY. (1992). Ecole et savoir dans les banlieues... et ailleurs. Paris : Colin.

Cicourel, A.V. (1979). La Sociologie cognitive. Paris : PUF. (1re édition 1973).

Clot, Y. (1999). La fonction psychologique du travail. Paris : PUF.

Clot, Y. (2013). Développer le métier : le collectif dans l'individu ? Communication au colloque ARIS Amiens.

Clot, Y. \& Lhuilier, D. (2012). Agir en clinique du travail. Toulouse : Eres.

Delcroix, A., Forissier, T., \& Anciaux, F. (2013). Vers un cadre d'analyse opérationnel des phénomènes de contextualisation didactique. Dans F. Anciaux, T. Forissier et LF. Prudent, Contextualisations didactiques (pp. 141-188). Paris : L'harmattan. 
eJRIEPS Numéro Spécial n¹ Avril 2018

Dolz, J., \& Tupin, F. (2011). La notion de situation dans l'étude des phénomènes d'enseignement et d'apprentissage des langues : vers une perspective sociodidactique. Recherches en éducation, 12, 82-96.

Giddens, A., (1987). La Constitution de la société-Éléments de la théorie de la structuration. Paris : PUF. (Édition originale : 1984).

Kherroubi, M., \& Rochex, J.-Y. (2004). La recherche en éducation et les ZEP en France. 2. Apprentissages et exercice professionnel en ZEP : résultats, analyses, interprétations. Revue française de pédagogie, 146, 115-190.

Lahire, B. (1996). La variation des échelles de contexte dans les sciences sociales. Remarques épistémologiques. Annales histoire, sciences sociales, 51(2), 381-407.

Lahire, B. (1998). L'homme pluriel. Paris : Nathan.

Lahire, B. (2008). Cadres sociaux de l'action et dispositions incorporées. Communication au colloque ARIS, Rodez.

Lahire, B. (2012). Monde pluriel. Paris : Seuil.

Marcel, J. F. (2002). Le concept de contextualisation : un instrument pour l'étude des pratiques enseignantes. Revue française de pédagogie, 138, 103-113.

Marlot, C. et Toullec-Théry, M. (2011). Caractérisation didactique des gestes de l'aide ordinaire à l'école élémentaire : une étude comparative de deux cas didactiques en mathématiques. Éducation \& didactique, 5 (3), 7-32.

Monnier, N. \& Amade-Escot, C. (2009). L'activité didactique empêchée : outil d'intelligibilité de la pratique enseignante en milieu difficile, Revue française de pédagogie, 168, 59-73.

Raynaud, D. (2006). Le contexte est-il un concept légitime de l'explication sociologique? L'année sociologique, 56(2).

Rochex, JY. (1995). Enseignants en banlieue ou enseignants de banlieue ? Dans Elisabeth Bautier (dir.), Travailler en banlieue. Paris : L'harmattan.

Rochex, J.-Y., \& Crinon, J. (2011). La construction des inégalités scolaires. Rennes : PUR.

Sensevy, G. (2006). L'action didactique. Eléments de théorisation. Revue suisse des sciences de l'éducation, 28(2), 205-225.

Sensevy, G. (2009). Jeux d'apprentissage, mésogénèse, chronogénèse. Une approche du travail de régulation du professeur. Intervention au séminaire de recherche du 
eJRIEPS Numéro Spécial n¹ Avril 2018

GRIAPS (groupe de recherche sur l'intervention en activités physiques et sportives), le 5 février 2009.

Tupin, F., \& Sauvage Luntadi, L. (2010). La situation professionnelle comme traduction du rapport dialectique entre acteurs et contextes. Actes du congrès de l'Actualité de la recherche en éducation et en formation (AREF), Université de Genève, septembre 2010.

Van Zanten, A., (2001). L'école de la périphérie. Scolarité et ségrégation en banlieue. Paris : PUF. 